\title{
Histological Analysis of the Growth of the Mandibular Condyle in the Rhesus Monkey (Macaca mulatta) 1
}

\author{
DAVID S. CARLSON, ${ }^{2}$ JAMES A. MCNAMARA, JR. ${ }^{2.3}$ AND DOUGLAS H. JAUL ${ }^{2.4}$ \\ ${ }^{2}$ Center for Human Growth and Development, ${ }^{3}$ Department of Anatomy, ${ }^{4}$ School \\ of Dentistry, The University of Michigan, Ann Arbor, Michigan 48109
}

\begin{abstract}
Qualitative and quantitative data on the growth of the mandibular condyle in the rhesus monkey (Macaca mulatta) are limited. The purpose of this investigation was to provide such data, with emphasis on variation in the size of the cartilaginous layers in the condyle and on condylar growth at five maturational levels (i.e., neonate, infant, juvenile, adolescent and young adult).

Two regions of the mandibular condyle, the articular tissue and the prechondroblastic-chondroblastic (growth) layer, were examined histologically in 38 rhesus monkeys. The absolute area of the articular layer increased dramatically from the neonatal through the juvenile age groups and then decreased gradually through the adult group. When the absolute values were expressed relative to condylar size, the first three maturational levels shared a common trend of increasing growth of the articular layer, with a cessation of growth in this tissue occurring during the adolescent period. This variation in articular layer tissue is probably the result of progressive alteration in the function of the temporomandibular joint.
\end{abstract}

The size of the prechondroblastic-chondroblastic (growth) cartilage increased dramatically between the neonatal and juvenile age groups, and subsequently decreased in older age groups. The relative thickness of the prechondroblasticchondroblastic cartilage reached its peak within the infant and juvenile levels, being greatest in the posterior region among the infants and in the postero-superior region among the juveniles. This corresponds to previous investigations which have shown that greater vertical growth of the rhesus monkey mandible occurs during the infant period, while the direction of mandibular growth is more horizontal in subsequent age groups.

The rhesus monkey (Macaca mulatta) remains the most commonly used nonhuman primate model for craniofacial growth in man. Most studies of facial growth in primates, however, involve experimental intervention and are characterized by a limited number of both experimental and control animals. Normal variation, especially during the growth process, is impossible to ascertain reliably using this approach. Thus, conclusions on normal craniofacial growth in monkeys from experimental studies may remain open to question (Carlsson and Öberg, '74).

Several recent studies document normal growth and development of the craniofacial complex in the rhesus monkey (Duterloo and Enlow, '70; Elgoyhen et al., '72), with particular reference to the maxillary complex
(McNamara et al., '76) and the mandible (Turpin, '68; McNamara and Graber, '75). Detailed descriptions of the normal microanatomy and postnatal growth of the temporomandibular joint in $M$. mulatta, however, are limited primarily to unpublished master's theses (Zielinski, '65; Zimmermann, '71) or to experimental investigations with few control animals (e.g., Hiniker and Ramfjord, '66; Cutler, '68; Norwich, '69; Stöckli and Willert, '71).

The purpose of this investigation was to provide a quantitative description of the normal microanatomy and growth of the tissues of the mandibular condyle from the neonate through

\footnotetext{
Accepted July $30, ' 77$.

1 This research was supported by the National Institute of Dental Research, NIH Research Grant DE 03610 and Post-Doctoral Research Fellowahip DE 05101.
} 
the young adult in the rhesus monkey. Emphasis was placed on the parameters of variation in the size of the cartilaginous layers in the condyle and on the direction of condylar growth at various age levels. These data are necessary for a more complete understanding of normal growth of the rhesus monkey, and are essential for accurate interpretation of facial growth following experimental intervention.

\section{Normal anatomy of the mandibular condyle}

The tissue of the mandibular condyle in mammals can be divided into two general layers: (1) an articular tissue layer and (2) a subarticular (growth) layer of cartilage (fig. 1). The subarticular layer can be further divided into prechondroblastic and chondroblastic zones. Each of these layers is present in the neonate and remains in the condyle through maturity (Carlsson and Öberg, '74). However, the absolute and relative size of each of the three layers may vary considerably depending on the overall rate of condylar growth and on the functional requirements placed on the temporomandibular joint. According to Cutler ('68) and Zimmermann ('71) the general features of condylar growth and morphology in the rhesus monkey and man are similar.

Articular tissue. The articular layer consists of a dense fibroelastic connective tissue whose collagen fibers are oriented parallel to the articular surface. It is largely avascular, particularly in its central region. There are only a few scattered fibroblasts present in the articular layer, which apparently function primarily in its maintenance rather than in the actual growth process (Kanouse et al., '69). The articular tissue varies in thickness along the condylar head, usually increasing in thickness posteriorly. It is continuous with the fibrous periosteum along the neck of the condyle (Wright and Moffett, '74).

Prechondroblastic (proliferative) zone. Immediately inferior to the articular layer, the outer portion of the proliferative zone is composed of undifferentiated mesenchymal cells within a basophilic ground substance. The embryonic cells differentiate into chondrocytes, appearing as densely packed, spindle-shaped cells which increase in size and become increasingly more separated due to continued production of intercellular matrix within the lighter-stained inner region of the prolifera- tive zone. Autoradiographic studies in the rat (Blackwood, '66; Petrovic et al., '75) and in the monkey (Kanouse et al., '69; Joondeph, '72) demonstrate that the outer portion of the prechondroblastic zone is the principal site of cell proliferation. As noted by Wright and Moffett ('74), the proliferative zone is continuous with the inner osteogenic portion of the periosteal membrane along the condylar neck.

Chondroblastic (hypertrophic) zone. The chondroblastic zone contains larger, spherical, maturing chondrocytes arranged in an apparently random fashion. Each cell is surrounded by a basophilic halo (territorial matrix). In growing animals, these cells hypertrophy, the nuclei become pyknotic, and the cytoplasm is increasingly evacuated as the cells are encroached upon the endosteal surface of the condyle. The intercellular matrix begins to mineralize within the innermost three to five levels of hypertrophying cells, and it is subsequently eroded by chondroclastic activity (Durkin et al., '73). Osteogenesis takes place at the junction of the hypertrophic zone and the endosteal surface either by endochondral ossification (Blackwood, '66; Wright and Moffett, '75) or, as suggested by Durkin and co-workers (Durkin et al., '73), by means of a special type of intramembranous bone formation.

\section{MATERIALS AND METHODS}

Thirty-eight rhesus monkeys (Macaca mulatta) from the primate colony of the Center for Human Growth and Development, The University of Michigan, were divided into five maturational levels for analysis. Animals from the first age group, neonate $(n=3)$, were obtained immediately post-partum. The infant monkeys ( $n=6$ ) had a complete deciduous dentition at the time of death, and ranged in known age from 6 to 12 months. The remaining three groups were delimited on the basis of dentitional development (Hurme and van Wagenen, '53, '61). Juveniles $(n=14)$ were characterized by a complete deciduous dentition and had their first permanent molars in full occlusion. These monkeys ranged in age from 18 to 30 months. The adolescent animals ( $n=8$ ) had a full permanent dentition, with the exception of unerupted third molars and were four to five years of age. The animals in the young adult $(n=7)$ group possessed complete permanent dentitions, including fully 

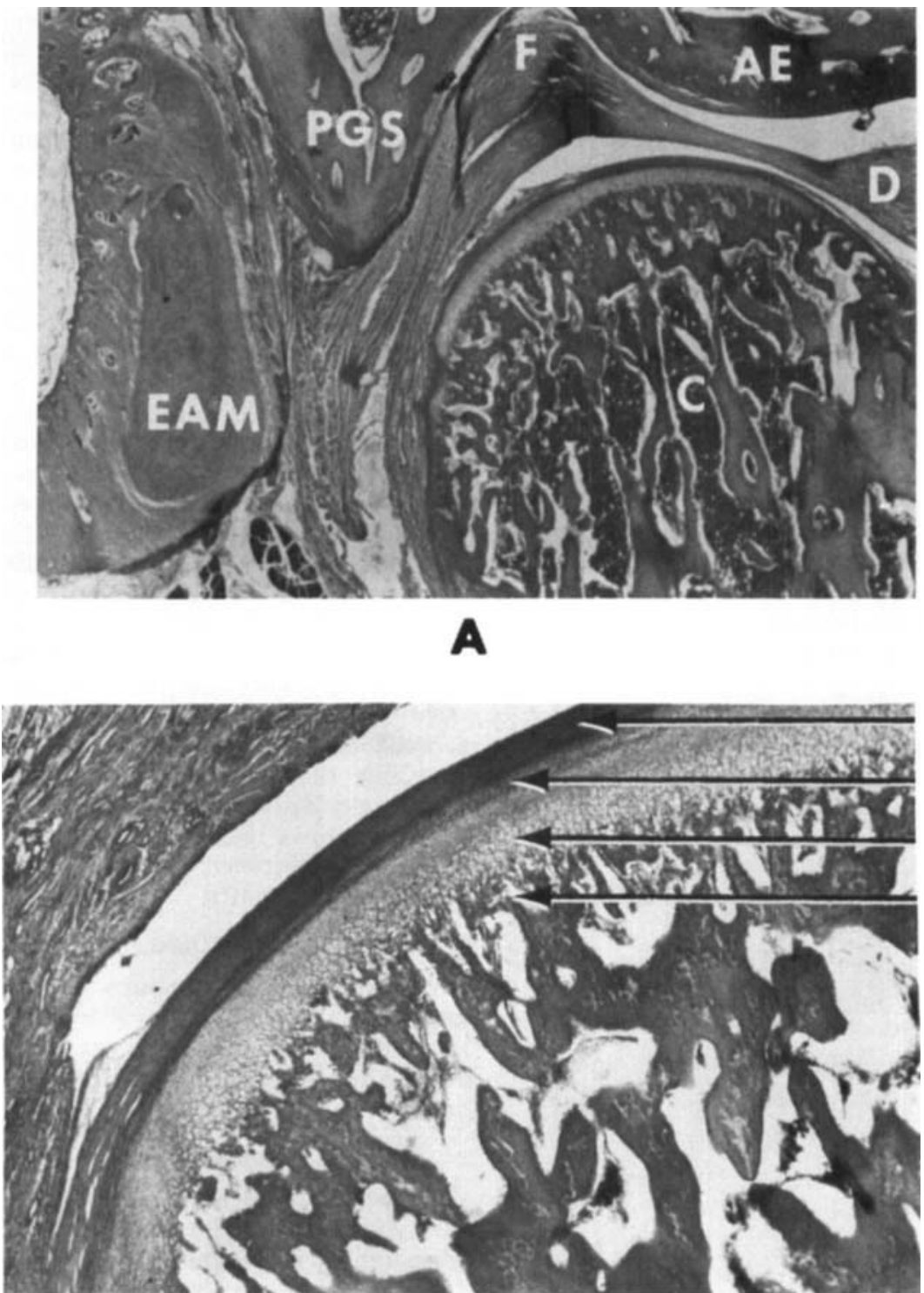

Fig. 1A General view of the right temporomandibular joint in juvenile Macaca mulatta, oriented according to the occlusal plane. External acoustic meatus (EAM), post-glenoid spine (PGS), mandibular condyle (C), articular disc $(D)$, articular eminence $(A E)$, mandibular fossa $(F)$. Hematoxylin and eosin $(\times 8)$, sagittal section.

B The zones of the condylar cartilage: $(A)$ articular zone; $(B)$ prechondroblastic (proliferative) zone; (C) chondroblastic zone (zone of maturation and hypertrophy); (D) region of bone deposition. Hematoxylin and eosin, sagittal section. $\times 40$.

erupted third molars, and were seven or more years of age.

A lateral cephalogram of each monkey was obtained immediately prior to death. All ra- diographs were taken, using a cephalostat especially designed for the laboratory monkey, at a distance of $155 \mathrm{~cm}$ (Elgoyhen et al., '72; McNamara, '72). 
Following perfusion with saline and phosphate-buffered formalin the right temporomandibular joint and its surrounding tissues were removed and placed in neutral buffered formalin. The tissue block was washed and decalcified in formic acid, embedded in either celloidin or paraffin and sectioned serially in the sagittal plane at 10 to 20 micrometers. Three midline sections were selected for study and stained with hemotoxylin and eosin. Histological sections were magnified $(\times 10)$ and microprojected $(\times 4.5)$ onto a flat surface from a distance of nine feet. Tracings were made of the temporomandibular joint structures, emphasizing the articular tissue and the prechondroblastic-chondroblastic cartilage layer within the mandibular condyle. No attempt was made to delineate the prechondroblastic zone from the chondroblastic zone since the gradual nature of their transformation made it difficult to identify a single boundary between these two regions.

\section{Measurements (fig. 2)}

Maximum length of the mandibular condyle was measured perpendicular to the posterior border of the ramus on the lateral cephalograms. The total areas occupied by the artic-

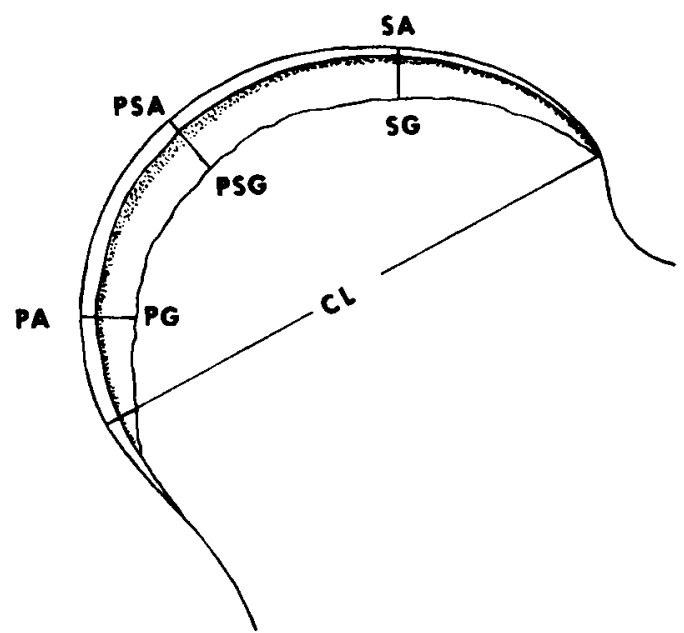

Fig. 2 Linear measurements recorded for the mandibular condyle. The orientation is the same as in figure 1. Maximum condylar length (CL) was measured perpendicular to the posterior border of the ramus on lateral cephalograms. Thicknesses of the articular tissue and prechondroblasticchondroblastic (growth) cartilage were measured perpendicular to the articular surface superiorly (SA, superior articular tissue; $S G$, superior growth cartilage), postero-superiorly (PSA, postero-superior articular tissue, $P S G$, posterosuperior growth cartilage), and posteriorly (PA, posterior articular tissue; $P G$, posterior growth cartilage). ular tissue and by the prechondroblastic-chondroblastic cartilage were determined from the tracings of each histological section using a compensating polar planimeter. Thicknesses of the layers were measured perpendicular to the articular surface at the superior, posterosuperior, and posterior regions around the circumference of the condyle. In order to account for variation in tissue area and thickness due to general body size, area measurements were divided by condylar length, squared, and thickness values were divided by condylar length.

Observations were recorded for each of the three midline sections per condyle and were then averaged for statistical evaluation. Means and standard deviations were determined to evaluate variation within each maturational level. One-way analysis of variance, non-simultaneous two-sample F-tests and two-sample Student's $t$-tests were computed to determine the significance of intergroup differences for this sample.

One-half of the total sample $(n=19)$ was retraced and remeasured independently to determine the error of the method. A twotailed $t$-test failed to demonstrate any significant differences between the means of the original and retraced subsamples for any of the 17 measurements.

\section{FINDINGS}

Summary statistics for each variable are reported by maturational level in table 1 . The significance of mean differences and variation between maturational levels are reported in table 2.

\section{Condylar length}

The overall size of the condyle increased greatly with age, and exhibited relatively little variation in length between individuals within the same age level (fig. 3). The greatest increase in condylar length took place between juvenile and adolescent groups (38\%), followed by the change between the adolescent and adult maturational levels (31\%). The differences among the group means were significant $(P<0.01)$.

\section{Articular tissue}

The absolute area of the articular layer increased from the neonate through the juvenile age groups $(\mathrm{P}<0.01)$. After reaching a mean peak of 529.6 square micrometers in the juvenile group, the articular layer decreased 


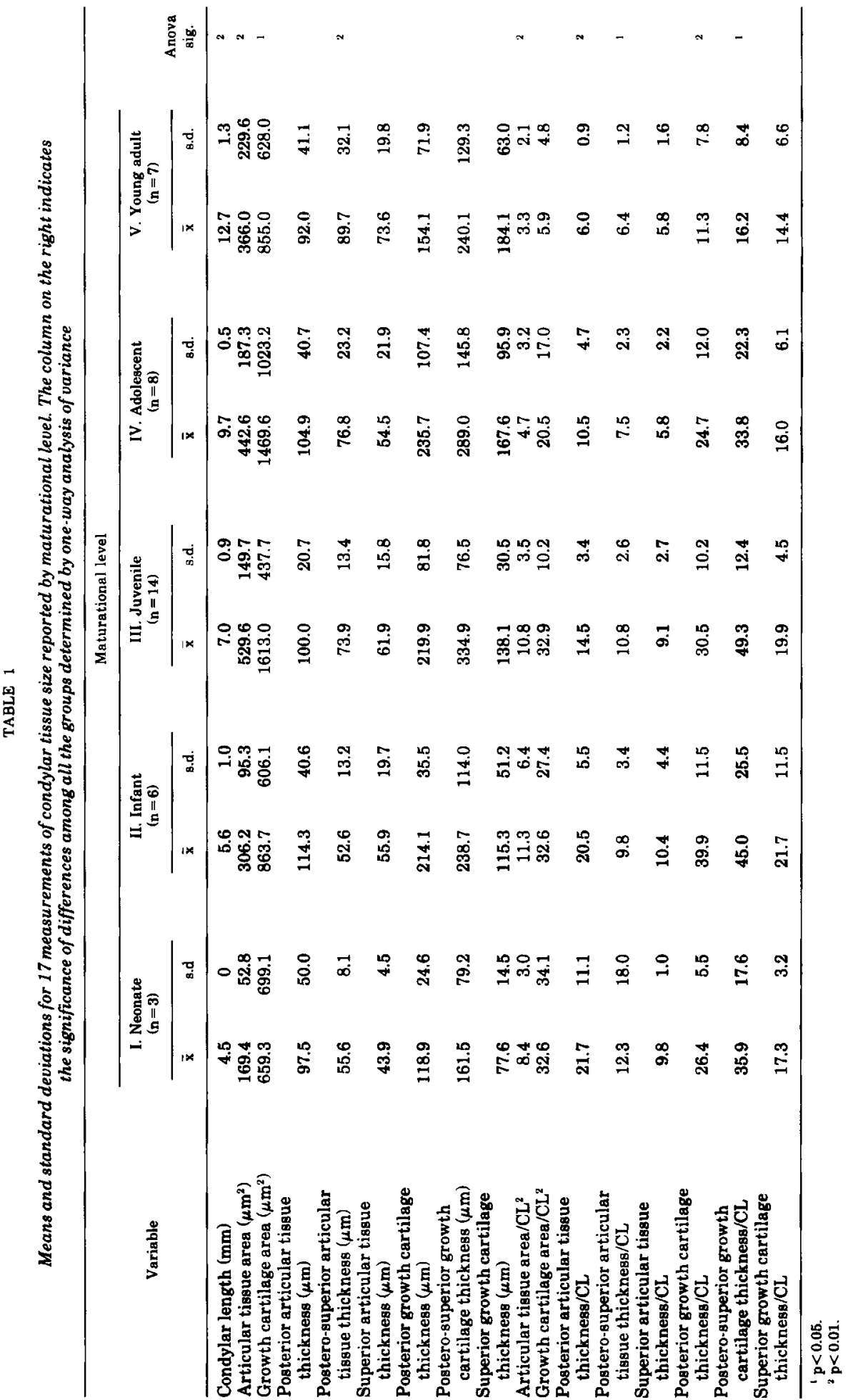


TABLE 2

Non-simultaneous significance levels for the two-tailed $t$ test $(\mathrm{t})$ testing equality of means between maturational groups, and two sample $F$-test $(\mathbf{F})$ testing equality of variance between maturational levels

\begin{tabular}{|c|c|c|c|c|c|c|c|c|c|c|}
\hline \multirow{2}{*}{ Variable } & \multicolumn{10}{|c|}{ Maturational level pair } \\
\hline & I-II & I-III & I-IV & I.V & II-III & II-IV & II-V & III-IV & III-V & IV.V \\
\hline Condylar length & $\mathbf{t}^{2}$ & $t^{2}$ & $t^{2}$ & $t^{2}$ & $t^{2}$ & $t^{2}$ & $t^{2}$ & $t^{2}$ & $t^{2}$ & $t^{2}$ \\
\hline Articular tissue area & $t^{1}$ & $t^{2}$ & $t^{1}$ & $F^{1}$ & $t^{2}$ & - & $F^{1}$ & - & - & - \\
\hline $\begin{array}{l}\text { Growth cartilage area } \\
\text { Posterior articular tissue }\end{array}$ & - & $t^{2}$ & - & - & $t^{2}$ & - & - & $\mathrm{F}^{2}$ & $t^{2}$ & - \\
\hline $\begin{array}{l}\text { thickness } \\
\text { Postero-superior articular }\end{array}$ & - & $\mathbf{F}^{2}$ & - & - & - & - & - & $\mathbf{F}^{1}$ & $\mathbf{F}^{1}$ & - \\
\hline $\begin{array}{l}\text { tissue thickness } \\
\text { Superior articular tissue }\end{array}$ & - & $t^{1}$ & - & - & $\mathbf{t}^{2}$ & $t^{2}$ & $t,{ }^{1} F^{1}$ & $\mathbf{F}^{1}$ & $\mathbf{F}^{2}$ & - \\
\hline $\begin{array}{l}\text { thickness } \\
\text { Posterior growth cartilage }\end{array}$ & $F^{1}$ & - & $\mathbf{F}^{1}$ & $\mathbf{t},{ }^{1} \mathbf{F}^{1}$ & - & - & - & - & - & - \\
\hline $\begin{array}{l}\text { thickness } \\
\text { Postero-super. growth }\end{array}$ & $t^{2}$ & $t^{2}$ & $\mathbf{F}^{2}$ & - & $F^{1}$ & $\mathbf{F}^{2}$ & - & - & - & - \\
\hline $\begin{array}{l}\text { cartilage thickness } \\
\text { Superior growth cartilage }\end{array}$ & - & $t^{2}$ & - & - & $t^{2}$ & - & - & $F^{1}$ & $\mathbf{F}^{1}$ & - \\
\hline thickness & - & $t^{2}$ & $F^{1}$ & $t,{ }^{1} F^{1}$ & - & - & - & $F^{1}$ & - & - \\
\hline Articular area/CL ${ }^{2}$ & - & - & - & $t^{i}$ & - & - & $t^{1} \mathbf{F}^{1}$ & $t^{2}$ & $t^{2}$ & - \\
\hline $\begin{array}{l}\text { Growth cartilage area/CL }{ }^{2} \\
\text { Posterior articular tissue }\end{array}$ & - & $\mathbf{F}^{1}$ & - & $F^{2}$ & $\mathbf{F}^{2}$ & - & $\mathrm{F}^{2}$ & - & $t^{2}$ & - \\
\hline $\begin{array}{l}\text { thickness/CL } \\
\text { Postero-super. artic. tissue }\end{array}$ & - & $\mathbf{t}^{1} \mathbf{F}^{2}$ & - & $F^{1}$ & $\mathbf{t}^{1}$ & $t^{2}$ & $\mathrm{t}^{2}{ }^{2} \mathrm{~F}^{\mathrm{I}}$ & - & $F^{1}$ & - \\
\hline $\begin{array}{l}\text { thickness/CL } \\
\text { Superior articular tissue }\end{array}$ & - & $t^{1}$ & - & $t^{2}$ & - & - & - & $t^{1}$ & $t^{1}$ & - \\
\hline $\begin{array}{l}\text { thickness/CL } \\
\text { Posterior growth cartilage }\end{array}$ & $\mathbf{F}^{1}$ & - & $t^{1}$ & $t^{2}$ & - & - & - & $t^{\prime}$ & $t^{1}$ & - \\
\hline $\begin{array}{l}\text { thickness/CL } \\
\text { Postero-super. growth cart. }\end{array}$ & - & - & - & $t^{1}$ & - & - & $t^{2}$ & - & $t^{2}$ & - \\
\hline $\begin{array}{l}\text { thickness/CL } \\
\text { Superior growth cartilage }\end{array}$ & - & - & - & - & $\mathrm{F}^{2}$ & - & $F^{1}$ & - & $\mathbf{t}^{2}$ & - \\
\hline thickness/CL & - & - & - & - & $F^{2}$ & - & - & - & - & - \\
\hline
\end{tabular}

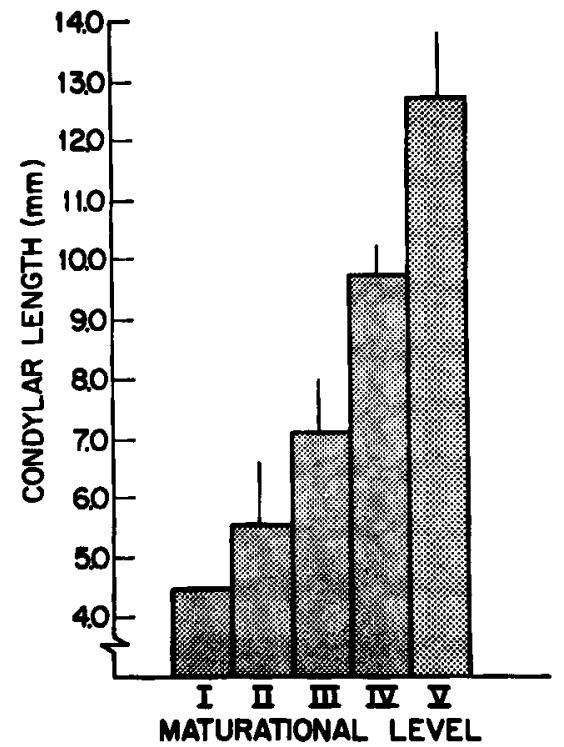

Fig. 3 Bar graph demonstrating means and standard deviations, in condylar length by maturational level: $I$, neonate; II, infant; III, juvenile; IV, adolescent; V, young adult. gradually in absolute area through the adult level (fig. 4A).

A slightly different pattern of change in the articular tissue emerged once the absolute values were divided by condylar size $\left(C L^{2}\right)$. Although the mean changes between the younger age groups were less marked, the variability within each age level was increased (fig. 4B). There was a small increase in area relative to condylar size between the neonates and infants, and a slightly greater decrease in relative area from the juvenile to the adult levels. According to this pattern, the first three maturational levels shared a common trend of increasing growth of the articular layer relative to condylar size, with a cessation of growth in the articular tissue occur. ring during the adolescent period.

The thickness of the articular layer varied considerably within individuals and between maturational levels. The superior and posterosuperior regions of the condyle exhibited a general trend between increasing thickness with age when absolute values were considered, while the posterior region appeared to 


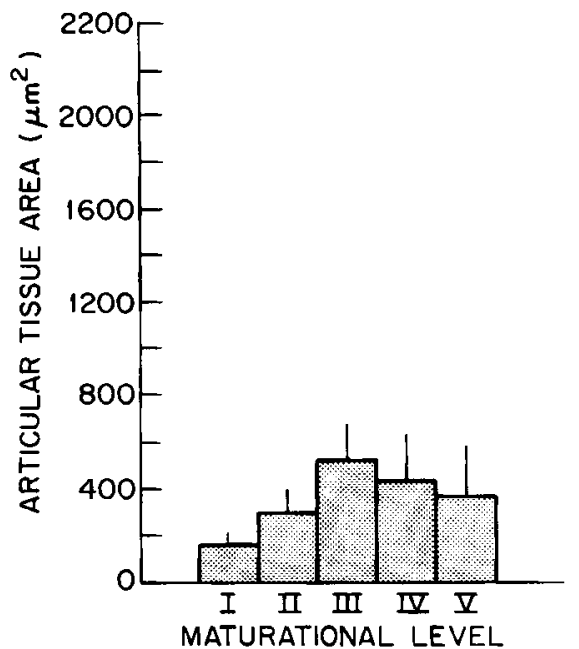

A

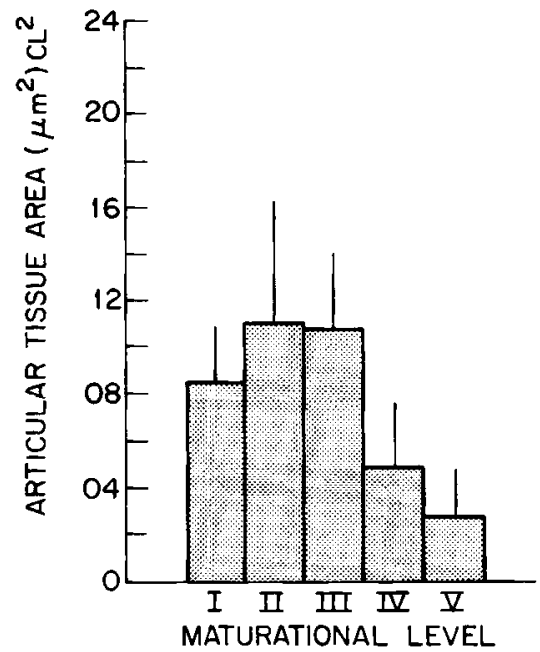

$B$

Fig. 4 Bar graphs indicating means and standard deviations for: (A) absolute values for the area occupied by the articular tissue, and (B) articular tissue area divided by $C L$.
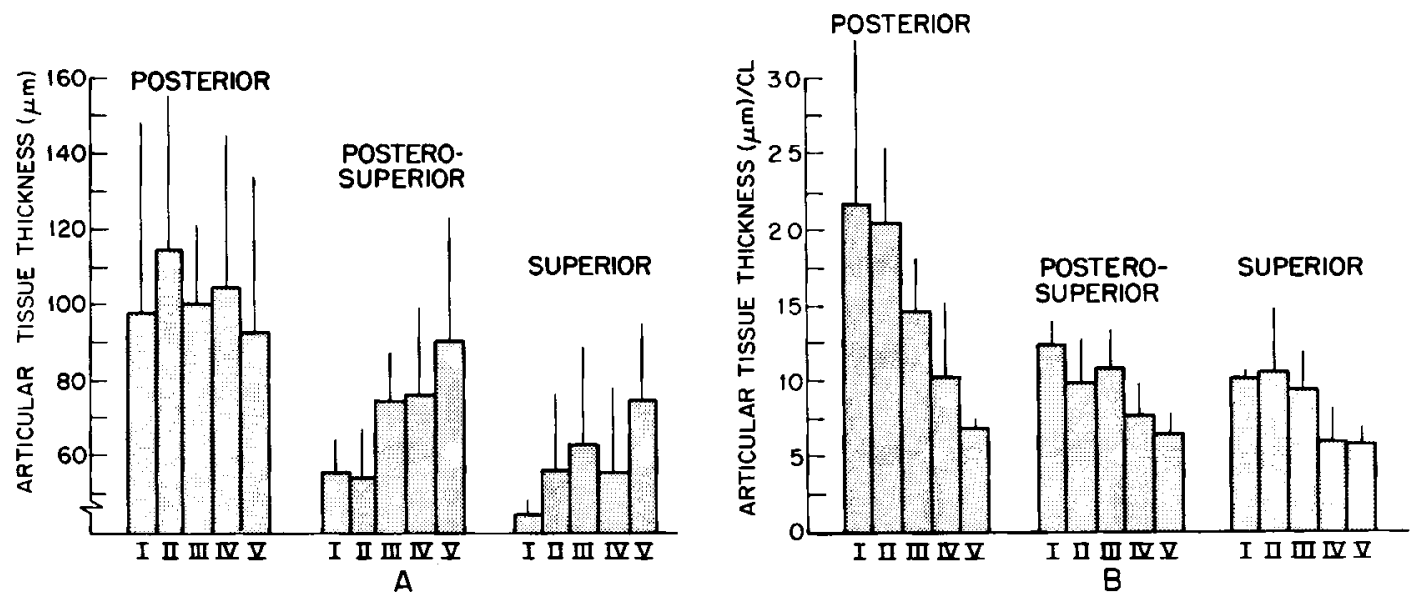

Fig. 5 Bar graphs of means and standard deviations for: (A) thickness of the articular tissue in the posterior, postero-superior, and superior regions of the condyle for each maturational level, and (B) thickness of the articular tissue in each region divided by condylar length.

change very little and apparently randomly between levels (fig. 5A). Expressing articular tissue thickness relative to the size of the condyle, however, revealed that there was a trend for a relative decrease in the thickness of the articular layer at all regions during growth (fig. 5B). These changes were most significant $(\mathrm{P}<0.01)$ at the postero-superior and posterior regions.

Evaluation of the relative thickness of each of the three regions of the condyle by age group indicated the general pattern of growth change in the articular layer (fig. 6). The posterior aspect of the condyle had the thickest articular tissue at every maturational level. However, its contribution to the total thick. ness of the articular layer decreased during growth from approximately $50 \%$ in neonates and infants to $36 \%$ in adults. The postero-superior and superior aspects increased in their relative contribution to the thickness of the articular layer with age; from approximately $30 \%$ and $25 \%$ in the neonates to $40 \%$ and $30 \%$ among adults respectively.

\section{Prechondroblastic-chondroblastic (Growth) cartilage}

The size of the growth cartilage, i.e., the 


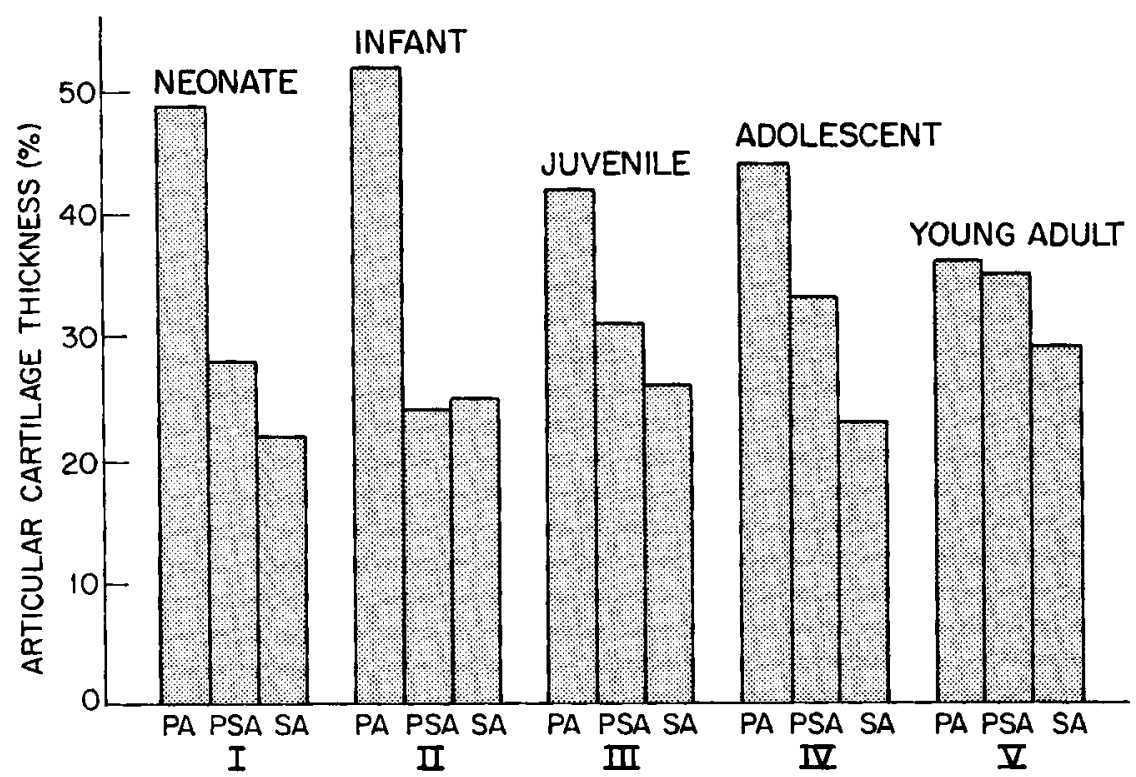

Fig. 6 Bar graphs indicating the percentage of the total articular tissue thickness accounted for by each of the three regions of the condyle at five maturational levels. Percentages were determined by dividing the thickness of each region of the articular layer (PA, PSA, and SA) by their sum, and multiplying by 100 .

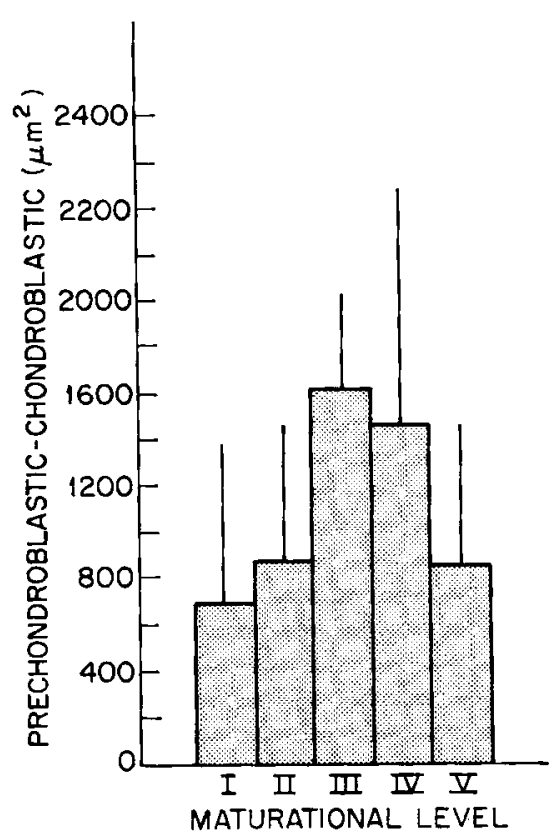

A

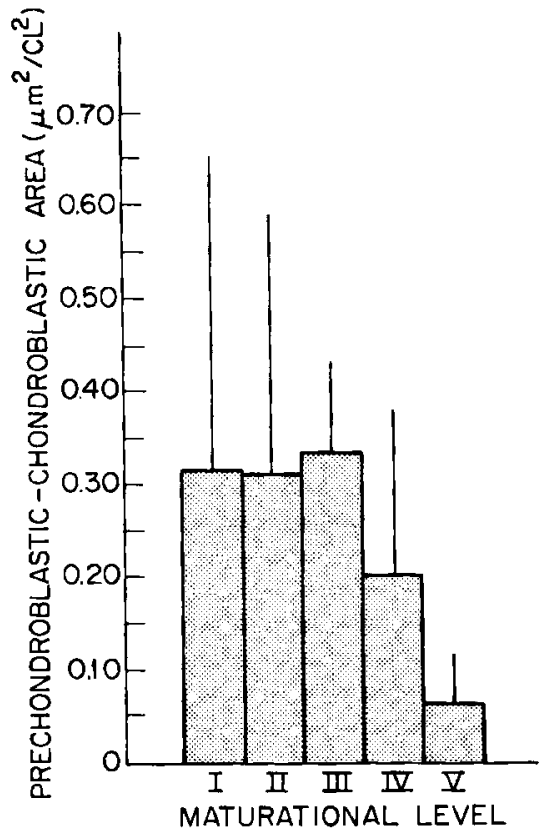

$B$

Fig. 7 Bar graphs indicating means and standard deviations for: (A) absolute values for the area occupied by the prechondroblastic-chondroblastic cartilage, and (B) prechondroblastic-chondroblastic cartilage area divided by $C L$. 
combined proliferative and hypertrophying zones, was considerably greater than that of the articular layer in every individual studied. As with the articular tissue, the total area of the prechondroblastic-chondroblastic cartilage for each histological section increased dramatically between the neonate and juvenile age groups, and subsequently decreased almost as abruptly through the adult level (fig. 7A). Despite considerable variation within each maturational level, there were significant differences between the mean for the juveniles and the means for most of the other groups for prechondroblastic-chondroblastic area.

Expressing the area of the prechondroblastic-chondroblastic layer as area per condylar length squared, a much different trend emerged (fig. 7B). The neonates, infants, and juveniles were all very similar with respect to relative area. As with the articular tissue, however, there was a sharp decrease in prechondroblastic-chondroblastic area relative to condylar size among the adolescents and adults.

The actual thickness of the prechondroblastic-chondroblastic cartilage varied according to two general patterns during growth. The growth layer at the superior aspect of the condyle increased in thickness in approximately

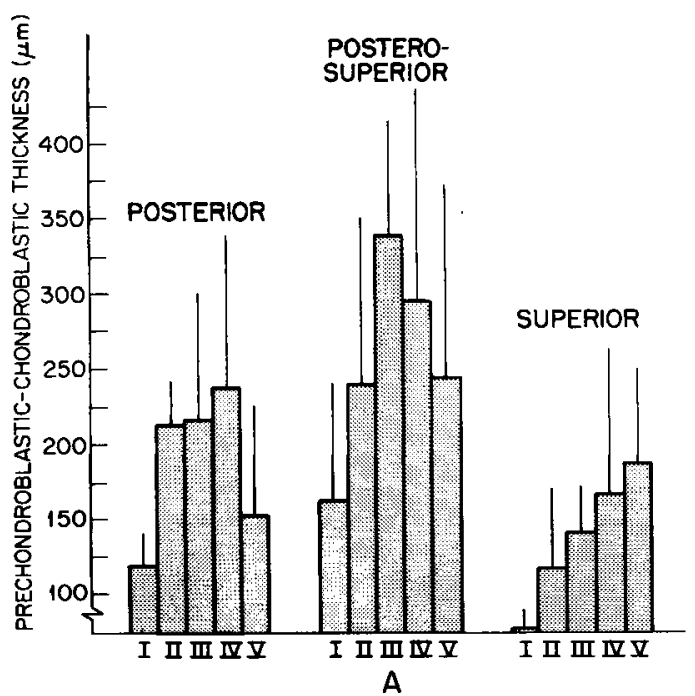

linear fashion with time (fig. 8A). Thicknesses in the postero-superior and posterior regions, on the other hand, tended to increase rapidly during the earlier ages, and then decrease at the later age levels. The cartilage in the postero-superior region reached its thickest point among the juveniles and became progressively thinner through the adolescent and adult age groups. In the posterior region, the most dramatic increase in thickness (44.5\%) took place between the neonates and infants. The infant, juvenile, and adolescent maturational levels remained very similar in mean thickness, although increasing in variation within groups. Posterior thickness then declined sharply through the adult age group.

Expressed relative to the length of the mandibular condyle, each of the three regions exhibited similar patterns of variation in thickness during growth (fig. 8B). The superior region and posterior region reached their greatest relative thickness during the infant period, and subsequently declined gradually through the adult group. The postero-superior region became steadily thicker relative to condylar size through the adults. Only the postero-superior and posterior regions exhibited growth changes that were statistically significant, although the similarity of pattern for all regions of the condyle lends credence to the

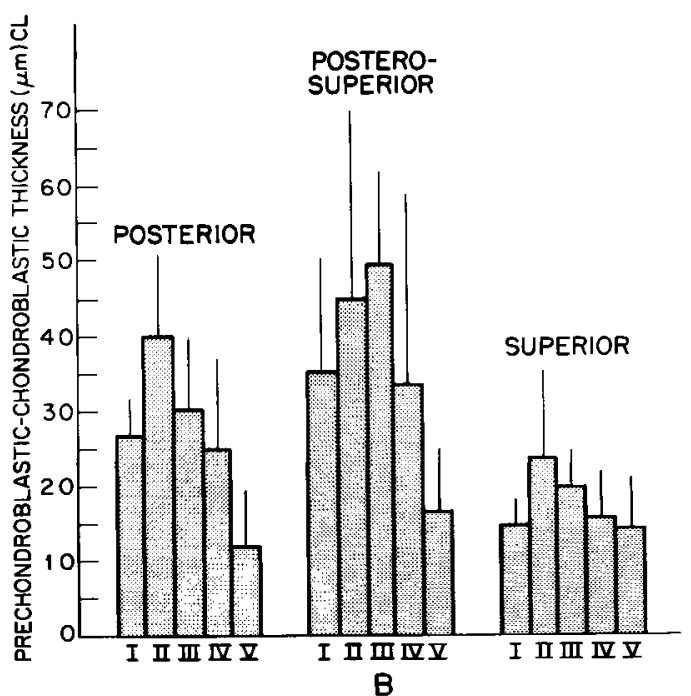

Fig. 8 Bar graphs of means and standard deviations for: (A) thickness of the prechondroblastic-chondroblastic cartilage in the posterior, postero-superior, and superior regions of the condyle for each maturational level, and (B) thickness of the prechondroblastic-chondroblastic cartilage in each region divided by condylar length. 


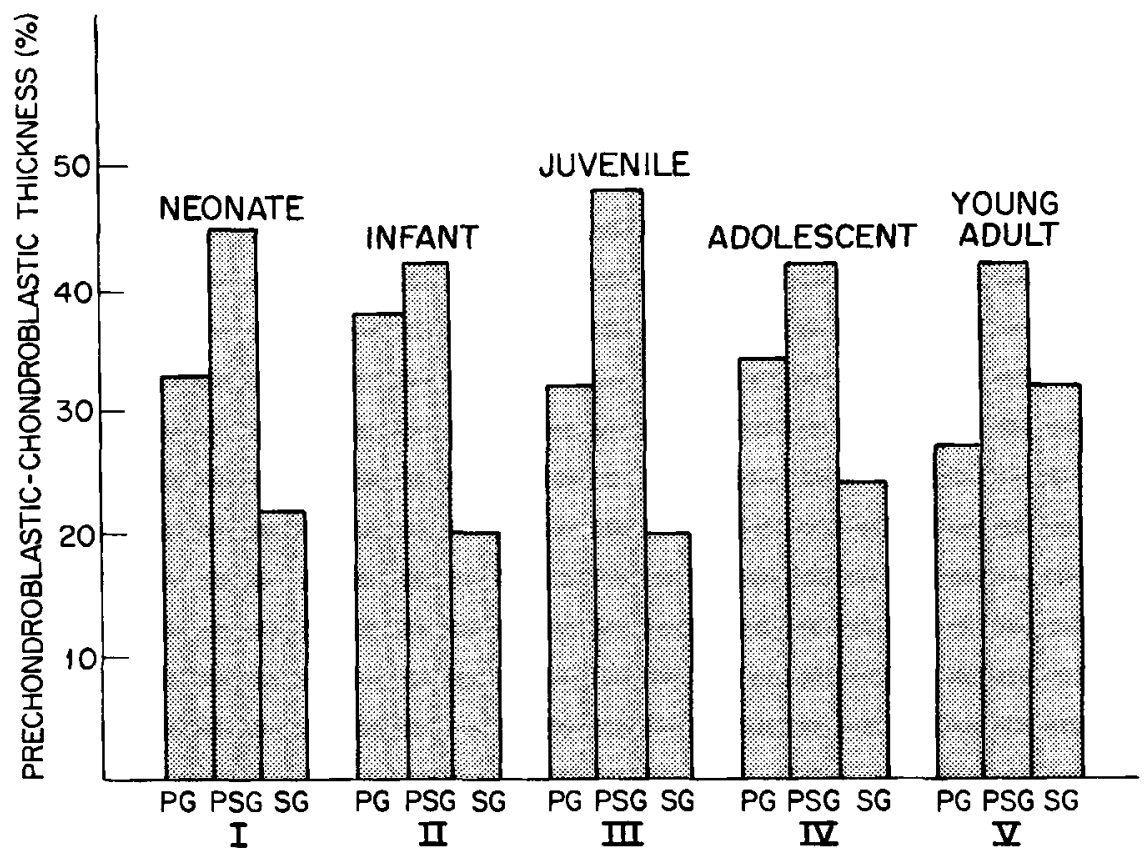

Fig. 9 Bar graphs indicating the percentage of the total prechondroblastic-chondroblastic cartilage thickness accounted for by each of the three regions of the condyle at five maturational levels. Percentages were determined by dividing the thickness of the prechondroblastic-chondroblastic layer of each region of the condyle (PG, PSG, and SG) by their sum, and multiplying by 100 .

changes seen during growth in the superior region as well.

The prechondroblastic-chondroblastic cartilage in the postero-superior aspect of the condyle remained both absolutely and relatively the thickest of the three regions from birth, becoming most prominent relative to the posterior and superior regions during the juvenile maturational level (fig. 9). It appeared from the histological sections that the proliferative zone accounted for a large part of the thickness in this region, especially during the juvenile period. The posterior region was the next thickest from birth through adolescence, reaching its thickest point relative to the rest of the condylar regions during the infant phase. The prechondroblastic-chondroblastic cartilage remained in evidence through the adult maturational level, and exhibited a more even tissue distribution among all three regions than previously at this time.

\section{Summary}

In summary, the growth patterns associated with the articular and prechondroblasticchondroblastic layers of the mandibular con- dyle of the rhesus monkey exhibited two major differences. Although similar patterns of change in relative areas were observed from the neonates through young adults, the layers differed with respect to relative thickness at specific regions of the condyle and at each maturational level. The articular tissue and growth cartilage remained roughly stable in relative area through the first three age levels, then decreased markedly through the adolescent and young adult periods. The greatest relative thickness of the articular tissue occurred in the posterior aspect of the neonatal condyle, and was characterized by a gradual decrease at all regions with age, with the greatest proportional decrease occurring in the posterior region. Relative thickness of the prechondroblastic-chondroblastic cartilage reached its peak within the infant and juvenile levels, being greatest in the posterior region among the infants and in the posterosuperior and posterior regions among the juveniles.

\section{DISCUSSION}

The results of this analysis are in general agreement with the description of temporo- 
mandibular joint growth in juvenile through adult macaques and the limited quantitative data for the thickness of the tissue layers reported by Zimmerman ('71). Specifically, Zimmermann stated that in monkeys between the ages of 18 and 24 months the articular tissue ranges from 40 to 90 micrometers thick, and is approximately 70 micrometers thick under the center of the articular disc. The means reported here range from about 62 to 105 micrometers for the articular layer as a whole, while the growth cartilage near the center of the disc has a mean thickness of approximately 74 micrometers for the same age level. The combined prechondroblastic-chondroblastic zone, according to Zimmermann, ranges from approximately 320 to 400 micrometers thick postero-superiorly and 225 micrometers superiorly in the 18- to 24-month group. The juveniles in the present study had means of approximately 335 micrometers and $138 \mathrm{mi}$ crometers for the postero-superior and superior thicknesses respectively.

\section{Articular tissue growth}

The factors which cause variation in thickness of the articular tissue are not entirely clear. However, the age-related trends described here do suggest some associations which may account for change in the articular tissue when viewed in light of the structure and function of the temporomandibular joint.

Although similar in many respects to articular cartilage elsewhere in the body, the articular tissue of the mandibular condyle possesses properties which are structurally and functionally unique. Beginning as a fibrous connective tissue continuous with the outer layer of the fibrous periosteum, the articular tissue of the mandibular condyle is transformed into fibrocartilage in those regions of greatest mechanical stress (Moffett et al., '66). Most articular cartilages function to absorb and alleviate compressive forces within joints. They are not well adapted to alleviating shearing forces. The articular surface of the mandibular condyle is subject to both compressive and shearing forces, however, since the condyle undergoes anterior and posterior translation during various phases of the masticatory cycle. Fibrous articular tissue is capable of withstanding both types of forces; and becomes more fibrocartilaginous in nature in the areas of maximal compressive forces, i.e., along the antero-superior surface of the condyle and the postero-inferior surface of the articular eminence in humans (Wright and Moffett, '74), while remaining fibrous in areas of maximal shearing stress.

Several researchers have noted that articular surfaces are active sites of growth and remodeling throughout life (Johnson, '59; Moffett et al., '66; Radin et al., '72; Carlsson and Öberg, '74). Articular tissues are highly adaptive to mechanical demands, with changes in function and associated mechanical stresses throughout life resulting in altered articular contour and size. Moffett ('66) noted, for example, that there is no correlation between articular remodeling and age in the temporomandibular joint and that the contour and thickness of the articular tissue is dependent primarily on jaw function and dental development. This observation is consistent with the fact that articular cartilage in other joints is thickest in areas of maximal stress (Johnson, '59). The mechanism by which articular tissue becomes thicker in specific areas is unclear, although it is known that articular cartilage becomes more active during use, and that its thickness increases as much as $10 \%$ due to increased water content of the surface layers in regions of greatest mechanical stress (Ekholm, '55; Ekholm and Norbach, '51).

The above information suggests that the variation in articular layer thickness within and between maturational levels reported here is probably the result of progressive alteration in the function of the temporomandibular joint. According to this hypothesis, the forces transmitted through the temporomandibular joint in younger monkeys should be greatest in the posterior region of the condyle, and should become more superiorly directed during maturation.

Supportive evidence for the hypothesis that variation in articular tissue thickness during growth in monkeys is due primarily to changes in the forces through the condyle comes from recent studies of temporomandibular joint anatomy and of the function of the muscles of mastication in young and adult rhesus monkeys. As in human infants, the temporomandibular joint in young monkeys is characterized by a shallow mandibular fossa and a relatively flat articular eminence ( $\mathrm{Zie}$ linski, '65; Biegert, '56). In humans, the mandibular fossa deepens and the articular eminence becomes more pronounced by two years of age (Wright and Moffett, '74). The articular eminence becomes only slightly more pro- 
nounced in the juvenile monkey, however, remaining relatively flat throughout life. Also unlike humans, the monkey temporomandibular joint is characterized by a prominent postglenoid spine (fig. 1) which, in young animals, undergoes bony resorption along its anterior surface (Zielinski, '65; Cutler, '68; Zimmermann, '71). According to Zimmermann ('71), the presence of a prominent post-glenoid spine and resorption along its anterior surface indicate that there may be contact between the condyle and the posterior region of the mandibular fossa in the monkey. The close proximity of the condyle and post-glenoid spine is most apparent in the younger animals, with the condyle becoming progressively more anteriorly located through adulthood (Zimmermann, '71). This positional change within the temporomandibular joint may be due to either an increase in the thickness of the posterior component of the articular disc, the influence of the dentition during the development of occlution, or both.

Recent electromyographic studies of mastication in monkeys also indicate distinct agerelated differences in function which could influence the direction and degree of forces being transmitted through the temporomandibular joint. Infant monkeys are characterized by a dominance of the anterior component of the temporalis muscle during the closing phase of the chewing stroke (McNamara, 74). Beginning with the juveniles, there is a trend for the masseter muscle and, to a lesser degree, the posterior component of the temporalis muscle to become more active during mandibular elevation. By the young adult period, the masseter muscle is clearly dominant during jaw closure, with the posterior fibers of the temporalis muscle being recruited only during forceful chewing.

In addition to changes in function, the morphology of the muscles of mastication undergoes progressive alteration during matura. tion. For example, the temporalis muscle in younger animals is relatively smaller and more fan-shaped than in adolescents and adults. Its anterior component originates more posteriorly along the cranial vault, expanding anteriorly concomitant with the development of the post-orbital bar in juvenile monkeys. The net effect of this morphologicalfunctional change is probably most apparent in the direction of muscle action during mastication. The direction of muscle pull in the temporalis muscle of young animals should be more posterior, producing the greatest amount of stress within the temporomandibular joint in the posterior aspect. During maturation, the anterior component of the temporalis muscle develops anteriorly and the masseter muscle becomes more dominant during mastication, thus increasing the anterior component of muscle action, resulting in relatively greater compressive forces on the antero-superior region of the condyle.

It is clear that the gross morphology of the temporomandibular joint and the structure and function of the muscles of mastication in the rhesus monkey undergo regular change with age. The data presented in this analysis indicate the additional presence of regional and age-related changes in the thickness of the articular tissue. It is probable that all of these changes in the rhesus monkey are causally interrelated. Alteration of the morphology of the temporomandibular joint and of the muscles of mastication, functional change in the mandibular elevators during mastication, and change in the relative thickness of the articular tissue all occur during the juvenile-adolescent maturational levels. It is also during this period that there is a mixed dentition, which may be an additional component of the age-related variation in the rhesus monkey masticatory complex.

\section{Prechondroblastic-chondroblastic cartilage growth}

Variation in the thickness of the prechondroblastic-chondroblastic cartilage between maturational levels and at different regions along the condyle can be accounted for by considering the growth of the mandible in the rhesus monkey and the role of the condylar cartilage during this growth process.

The prechondroblastic-chondroblastic cartilage is a principal site of adaptive growth. Recent experimental research demonstrates that the role of the growth cartilage within the condyle is to maintain the functional integrity of the temporomandibular joint during translation of the mandible downward and forward during craniofacial growth (Stöckli and Willett, '71; McNamara, '72, '73; Petrovic et al., '75). Furthermore, the apparently haphazard arrangement of the cells within the prechondroblastic and chondroblastic zones suggests that the condyle has the capac. ity for multidirectional growth and, thus, possesses the potential for relatively rapid adaptation to altered mandibular position or 
function. These data indicate, in fact, that the prechondroblastic - chondroblastic cartilage would be relatively isogonous in the absence of constraints placed upon it by extrinsic factors, such as biomechanical forces during jaw function, as well as by the developing dentition and altered occlusion. Thus, the way to explain variation in the thickness, i.e., activity, of the prechondroblastic-chondroblastic cartilage is to examine the growth of the maxillomandibular complex as a whole.

A recent study by Björk and Skieller ('72) has provided a detailed account of the rotation of the jaws which characterizes facial growth in humans. They noted that in humans there is normally a forward rotation of the maxillomandibular complex during growth, i.e., a posterior opening due to a decreased angulation of the occlusal plane relative to the nasionsella line. However backward rotation, i.e., a posterior closing due to an increased angulation of the occlusal plane, does occur in some individuals. Compensatory remodeling in both instances takes place along the inferior border of the mandible, principally in the symphyseal region and in the anterior portion of the corpus. Björk and Skieller further noted that the rate and direction of condylar growth are strongly correlated with the rotation of the mandible.

The geometry of the rotations described by Björk and Skieller and the compensatory changes at the condyles may not be immediately obvious. According to these authors, during forward rotation of the mandible, the corpus "pivots" about either the incisors or the premolars, probably as a result of a greater extent of eruption of the molars than the incisors, causing the occlusal plane to become more open posteriorly relative to the cranial base. This posterior opening of the occlusal plane causes the symphyseal region of the mandible to be translated anteriorly, resulting in a distal displacement of the condyle and stimulating growth of the condyle in a superior direction. During backward rotation, the mandible "pivots" about the molar region due to a greater eruption of the incisors relative to the premolars and molars, thus closing the occlusal plane posteriorly. This type of rotation translates that symphyseal region inferiorly and posteriorly, causing the condyle to be displaced anteriorly and stimulating posterior growth of the condyle. These data indicate that the growth of the condyle is intimately related to the alterations in its position within the temporomandibular joint, such that the rate and direction of condylar growth is largely compensatory to the translation of the con. dyle which is brought about by the rotation of the jaws.

Recent studies demonstrate that craniofacial growth in the rhesus monkey is also characterized by a rotation of the jaws. However, the direction of the rotation is less variable in the monkey than in humans. McNamara and Graber ('75) and McNamara et al. ('76) noted that the rhesus monkey exhibits only a forward rotation of the maxillo-mandibular complex during growth. Consistent with the findings of Björk and Skieller ('72), forward rotation in the rhesus monkey tends to be masked by greater bone growth and remodeling in the symphyseal region and along the anterior aspect of the inferior border of the mandible than along the posterior aspect, and by a greater vertical migration of the mandibular teeth posteriorly than anteriorly.

Forward rotation of the maxillo-mandibular complex during growth leads to a dominance of the anterior component of growth of the mandible in the rhesus monkey. Nevertheless, there is a regular pattern of variation in the growth of the condylar region which incorporates a vertical trend as well (McNamara and Graber, '75). Infant monkeys exhibit a trend toward greater vertical growth of the mandible, while maximum horizontal growth of the mandible occurs during the juvenile maturational level. This is manifested cephalometrically by a relatively greater posterior growth of the condylar region among the infants, and a relatively greater postero-superior growth during the juvenile period.

The results of this analysis support the data reported by McNamara and co-workers for craniofacial growth in $M$. mulatta when considered in light of the associations between the developing dentition, condyle, and rotations of the jaws reported by Björk and Skieller ('72). The tendency for greater vertical growth of the rhesus monkey mandible during the infant period is confirmed by the fact that the relative thickness of the posterior prechondroblastic-chondroblastic cartilage is greatest during this time, and that it gradually tapers off during successive age groups. The postero-superior region of the growth cartilage is thickest at every maturational level; however, it reaches its maximal relative thickness during the juvenile period. It is during this period that the forward rota- 
tion of the mandible in the rhesus monkey is greatest. Relative thickness of the cartilage in the postero-superior region and anterior growth of the mandible both gradually diminish during the adolescent and young adult maturational levels.

\section{CONCLUSION}

This study provides qualitative and quantitative data on the growth of the mandibular condyle from birth through young adulthood in rhesus monkeys. These data may be used as baseline values in comparison with histological data from experimental studies of craniofacial growth.

There are at least two major conclusions which can be drawn from this analysis.

(1) Despite considerable intragroup variation, it is clear that the rate and direction of growth of the articular tissue and of the prechondroblastic-chondroblastic layers of the mandibular condyle undergo relatively regular variation between maturational levels. Relative area of the condylar tissue layers is similar among the neonates, infants and juveniles, and decreases in the adolescent and young adult groups. Relative thickness of the articular tissue is greatest posteriorly in the neonate, becoming proportionately thinner in this region during growth. The rate of condylar growth in the prechondroblastic-chondroblastic layer is greatest among the infants and juveniles, with the infant condyle being characterized by relatively greater posterior growth and the juveniles being characterized by greater growth in a postero-superior direction.

(2) The associations between these data and other developmental aspects of craniofacial growth in the rhesus monkey suggest causal interrelationships which may account for the rate and direction of condylar growth. It is hypothesized that variation in the thickness of the articular tissue is due primarily to morphological and functional changes in the temporomandibular joint during growth. Finally, it is suggested that the rate and direction of growth of the prechondroblastic-chondroblastic cartilage of the condyle are largely a result of the normal rotation of the maxillo-mandibular complex and associated dental development during craniofacial growth.

\section{ACKNOWLEDGMENTS}

We gratefully acknowledge the technical assistance of M. Christine McBride and Rob- ert L. Wainwright, and the editorial assistance of Christine McCabe.

\section{LITERATURE CITED}

Biegert, J. 1956 Das Kiefergelenk der Primaten. Morph. Jb., $97: 249.404$.

Björk, A., and V. Skieller 1972 Facial development and tooth eruption: An implant study at the age of puberty. Amer. J. Orthod, 62: 339-383.

Blackwood, H. J. J. 1966 Adaptive changes in the mandibular joints with function. Dent. Clinics of North Amer., Nov.: 559-566.

Carlsson, G. E., and T. Öberg 1974 Remodeling of the temporomandibular joints. Oral Sci. Rev., 6: 53-86.

Cutler, B. S. 1968 Dentofacial Changes Produced by a Modified Milwaukee Brace in Macaca mulatta: A Roentgenographic and Histological Study. Unpublished Master's Thesis, Univ. of Washington, Seattle.

Durkin, J. F., J. D. Heeley and J. T. Irving 1973 The cartilage of the mandibular condyle. Oral Sci. Rev., 2: 29-99.

Duterloo, H. S, and D. H. Enlow 1970 A comparative study of cranial growth in Homo and Macaca. Am. J. Anat., 127: $357-368$.

Ekholm, R. 1955 Nutrition of articular cartilage. Acta Anat., 24: 329-338.

Ekholm, R., and B. Norback 1951 Relation between articular changes and function. Acta Orthop. Scandinav., 21: 81-98.

Elgoyhen, J. C., M. L. Riolo, L. W. Graber, R. E. Moyers and J. A. MeNamara, Jr. 1972 Craniofacial growth in juvenile Macaca mulatta: A cephalometric study. Am. J. Phys. Anthrop., 36. 369-376.

Hiniker, J. J., and S. P. Ramfjord 1966 Anterior displacement of the mandible in adult rhesus monkeys. J. Prosthet. Dent., 16: 503-512.

Johnson, L. C. 1959 Kinetics of osteoarthritis. Lab. Invest., 8: 1223-1241.

Joondeph, D. R. 1972 An autoradiographic study of the temporomandibular articulation in the growing Saimiri sciureus monkey. Amer. J. Orthod, 62: 272-286.

Kanouse, M. C., S. P. Ramfjord and C. E. Nasjleti 1969 Condylar growth on rhesus monkeys. J. Dent. Res., 48; 1171-1176.

McNamara, J. A., Jr. 1972 Neuromuscular and Skeletal Adaptations to Altered Orofacial Function. Center for Human Growth and Development, Ann Arbor, Michigan.

McNamara, J. A., Jr., T. G. Connelly, and M. C. McBride 1975 Histological studies of temporomandibular joint adaptations. In: Determinants of Mandibular Form and Growth. J. A. McNamara, ed. Center for Human Growth and Development, Ann Arbor, pp. 209-227.

MeNamara, J. A., Jr., and L. W. Graber 1975 Mandibular growth in the rhesus monkey (Macaca mulatta). Am. J. Phys. Anthrop., 42: 15-24.

McNamara, J. A., Jr., M. L. Riolo and D. H. Enlow 1976 Growth of the maxillary complex in the rhesus monkey (Macaca mulatta). Am. J. Phys. Anthrop., 44: 15-26.

Moffett, B. C., Jr. 1966 The morphogenesis of the temporomandibular joint. Amer. J. Orthod., 52: 401-415.

Moffett, B. C., Jr., L. C. Johnson, J. B. McCabe and H. C. Askew 1964 Articular remodeling in the adult human temporomandibular joint. Am. J. Anat., 115: 119-142.

Norwick, K. W. 1969 The Effect of Reciprocal Intermaxillary Forces (Class II) on the Growing Dentofacial Com. plex in the Macaca mulatta. Unpublished Master's Thesis, Univ. of Washington, Seattle.

Petrovic, A. G., A. J. Stutzmann and C. L. Oudet 1975 Control processes in the postnatal growth of the condylar 
cartilage of the mandible. In: Determinants of Mandibular Form and Growth. J. A. McNamara, Jr., ed. Center for Human Growth and Development, Ann Arbor, pp. 101. 153.

Radin, E. L., I. L. Paul and R. M. Rose 1972 Role of mechanical factors in pathogenesis of primary osteoarthritis. Lancet (March), 4: 519-521.

Stöckli, P. W., and H. G. Willert 1971 Tissue reactions in the temporomandibular joint resulting from anterior displacement of the mandible in the monkey. Amer. $J$. Orthod., 60: 142-155.

Turpin, D. L. 1968 Growth and remodeling of the mandi- ble in the Macaca mulatta monkey. Amer. J. Orthod., 54: 251-271.

Wright, D. M., and B. C. Moffett, Jr. 1974 The postnatal development of the human temporomandibular joint. Am. J. Anat., 141: 235-250.

Zielinski, D. E. 1955 A Study of the Normal Anatomy of the Masticatory Apparatus of Macaca mulatta. Unpublished Master's Thesis, Loyola Univ., Chicago.

Zimmermann, H. I. 1971 The Normal Growth and Remodeling of the Temporomandibular Joint of Macaca mulatta. Unpublished Master's Thesis, Univ. of Washing ton, Seattle. 